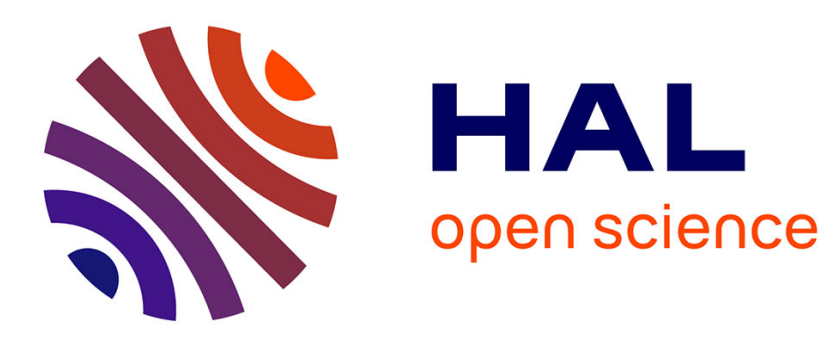

\title{
Disorder-Promoted Splitting in Quasiparticle Interference at Nesting Vectors
}

\author{
V. Stolyarov, V. Sheina, D. Khokhlov, S. Vlaic, S. Pons, H. Aubin, R.
} Akzyanov, A. Vasenko, T. Menshchikova, E. Chulkov, et al.

\section{- To cite this version:}

V. Stolyarov, V. Sheina, D. Khokhlov, S. Vlaic, S. Pons, et al.. Disorder-Promoted Splitting in Quasiparticle Interference at Nesting Vectors. Journal of Physical Chemistry Letters, 2021, 12 (12), pp.3127-3134. 10.1021/acs.jpclett.1c00462 . hal-03365704

\section{HAL Id: hal-03365704 https://hal.science/hal-03365704}

Submitted on 8 Oct 2021

HAL is a multi-disciplinary open access archive for the deposit and dissemination of scientific research documents, whether they are published or not. The documents may come from teaching and research institutions in France or abroad, or from public or private research centers.
L'archive ouverte pluridisciplinaire HAL, est destinée au dépôt et à la diffusion de documents scientifiques de niveau recherche, publiés ou non, émanant des établissements d'enseignement et de recherche français ou étrangers, des laboratoires publics ou privés. 


\section{Disorder-promoted splitting in quasiparticle interference at nesting vectors}

V. S. Stolyarov, ${ }^{*, \dagger, \uparrow}$ V.A. Sheina, ${ }^{\S, \ddagger}$ D. A. Khokhlov, ${ }^{\boldsymbol{\Phi}, \ddagger}, \|$ S. Vlaic, ${ }^{\dagger}$ S. Pons, ${ }^{\dagger}$

H. Aubin,,$^{\S, \dagger}$ R. S. Akzyanov, $,{ }^{\uparrow},\|\|$ A. S. Vasenko, ${ }^{\perp, \#}$ T. V. Menshchikova, ${ }^{@}$ E. V. Chulkov,,$^{\triangle} \perp$ A. A. Golubov, ${ }^{\nabla, \ddagger}$ T. Cren, ${ }^{\dagger \dagger}$ and D. Roditchev ${ }^{\dagger, \dagger+\ddagger}$

†Laboratoire de Physique et d'Étude des Matériaux (LPEM), UMR-8213, ESPCI Paris,

PSL Research University, CNRS, Sorbonne Université, 75005 Paris, France

$\ddagger T Q P S S$ Lab, Center for Photonics and $2 D$ Materials, Moscow Institute of Physics and Technology, Dolgoprudny, 141700 Moscow, Russia

9Dukhov Research Institute of Automatics (VNIIA), 127055 Moscow, Russia

$\S C 2 N$, UMR-9001, CNRS, Paris-Saclay Université, 91120 Palaiseau, France

|| Institute for Theoretical and Applied Electrodynamics, Russian Academy of Sciences, Moscow, 125412 Russia

$\perp$ National Research University Higher School of Economics, 101000 Moscow, Russia \#I.E. Tamm Department of Theoretical Physics, P.N. Lebedev Physical Institute, Russian Academy of Sciences, 119991 Moscow, Russia

@ Tomsk State University, 634050 Tomsk, Russia

$\triangle$ Donostia International Physics Center (DIPC), Paseo Manuel de Lardizabal 4, San Sebastián/Donostia, 20018 Basque Country, Spain

$\nabla$ Faculty of Science and Technology and MESA ${ }^{+}$Institute for Nanotechnology, University of Twente, 7500 AE Enschede, The Netherlands $\dagger † I N S P$, UMR-7588, Sorbonne Université, CNRS, 75005 Paris, France

E-mail: vasiliy.stoliarov@gmail.com 


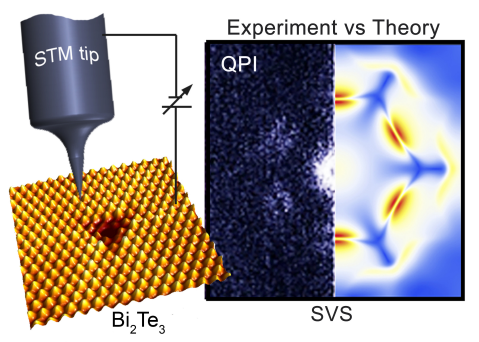

\begin{abstract}
Inelastic interactions of quantum systems with environment usually wash coherent effects out. In the case of Friedel oscillations the presence of disorder leads to a fast decay of the oscillation amplitude. Here we show both experimentally and theoretically that in the three-dimensional topological insulator $\mathrm{Bi}_{2} \mathrm{Te}_{3}$ there is a nesting-induced splitting of coherent scattering vectors which follows a peculiar evolution in energy. The effect becomes experimentally observable when the lifetime of quasiparticles shortens, due to disorder. The amplitude of the splitting allows evaluating the lifetime of the electrons. A similar phenomenon should be observed in any system with a well-defined scattering vector regardless of its topological properties.
\end{abstract}

Predicted a long ago, recently discovered three-dimensional topological insulators (TIs) ${ }^{1-7}$ are characterized by conducting surface states with the linear dispersion, Dirac cones, evolving in the bulk gap. In real materials, the Dirac cones are often regular only close to their origin, the Dirac point (DP). Both theories and experiments showed that far from the Dirac point, the circular shape of the constant energy contour evolves into a hexagon and then to a snowflake with sharp tips extending along six crystallographic directions. ${ }^{8-10}$ The warping is present in $\mathrm{Bi}_{2} \mathrm{Se}_{3},{ }^{11-13} \mathrm{~Pb}(\mathrm{Bi}, \mathrm{Sb})_{2} \mathrm{Te}_{4}{ }^{14}$ and other TI materials. ${ }^{10,15}$

The spin texture of Dirac quasiparticles in TIs is peculiar, due to momentum $\mathbf{k}$ coupling to spin $\sigma$; it is subject of intense studies. ${ }^{16}$ Close to impurities, the elastic electron scattering from the state $|\mathbf{k}, \sigma\rangle$ to the state $\left|\mathbf{k}^{\prime}, \sigma^{\prime}\right\rangle$ causes the interference between the incident and reflected waves with the wave vector $\mathbf{q}=\mathbf{k}-\mathbf{k}^{\prime}$ and the amplitude determined by the corresponding scattering matrix element. This gives rise to spatial variations of the local density of states (LDOS $)^{17}$ which can be probed by Scanning Tunneling Microscopy and Spectroscopy 
(STM/STS). The Fourier-transformed tunneling conductance $d I / d V(x, y)$ STS maps display quasiparticle interference (QPI) patterns and reveal characteristic scattering vectors. ${ }^{18,19}$

In search for specific scattering rules in three-dimensional TIs, the QPI patterns were studied around individual defects, ${ }^{20-23}$ steps, ${ }^{9,20,24}$ trappings, ${ }^{25}$ composition variations, ${ }^{26}$ etc. ${ }^{27}$ These experiments concluded that the backscattering from nonmagnetic impurities is forbidden, as theoretically expected, due to the opposite spin configurations between initial and final states. ${ }^{28-30}$

In the case of scattering on single defects in $\mathrm{Bi}_{2} \mathrm{Te}_{3}$, the reported QPI patterns consist of six scattering vectors along $\Gamma-\mathrm{M}$ directions. ${ }^{22}$ Though, numerical calculations ${ }^{28,31}$ expected comparable weights of scattering vectors along $\Gamma-\mathrm{K}$ and $\Gamma-\mathrm{M}$ directions. These calculations neglected however the actual form of the scattering potential. In $\mathrm{Bi}_{2} \mathrm{Se}_{3}$, for instance, Sevacancies are native surface defects ${ }^{32}$ that are charged ${ }^{33}$ and induce the charge density located in vertices of the equilateral triangle of a lateralsize $\sim 15-20 \AA,^{34}$ that is comparable with the wavelengths $2 \pi /|\mathbf{q}|$ of considered Friedel oscillations. The effects related to the finite quasiparticle lifetime were not considered yet. In experiments, the QPI patterns were measured around individual impurities chosen away from other defects. ${ }^{22}$ In models, the finite lifetime was introduced only to avoid divergences in calculations. ${ }^{28-30}$ However, most of macroscopic electronic properties are averaged over a large collection of impurities, often considered in theories as a homogeneous disorder.

In the present paper we study the QPI from a collection of Te-vacancies - native surface defects in $\mathrm{Bi}_{2} \mathrm{Te}_{3}$. Using STM we focus on large areas where hundreds of Te-vacancies ${ }^{35}$ are present, dense enough to enable the overlap of Friedel oscillations from neighbouring scatters. By analysing the resulted QPI patterns, we reveal a peculiar splitting of scattering vectors in the energy window 60-120 meV above the Fermi level, that vanishes at lower and higher energies. We evaluate the Green's function of the system with a scatter numerically in the framework of T-matrix approach ${ }^{29,36-38}$ (see Supplementary Eq. 4). The calculations show that the observed splitting occurs at energies at which parts of the constant energy 
a

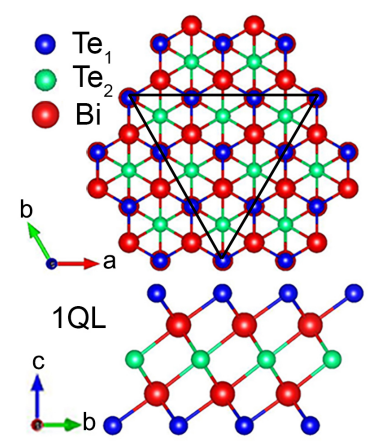

d

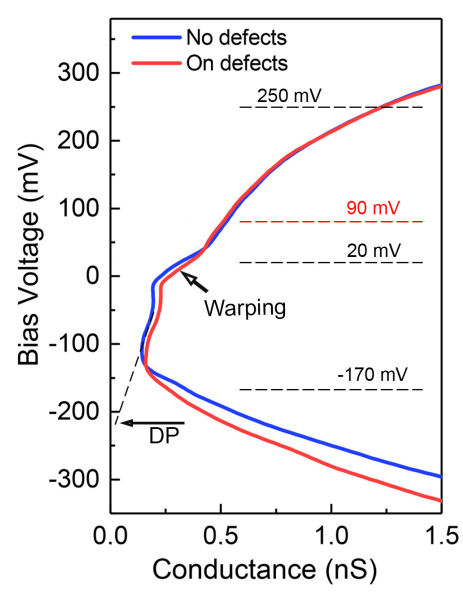

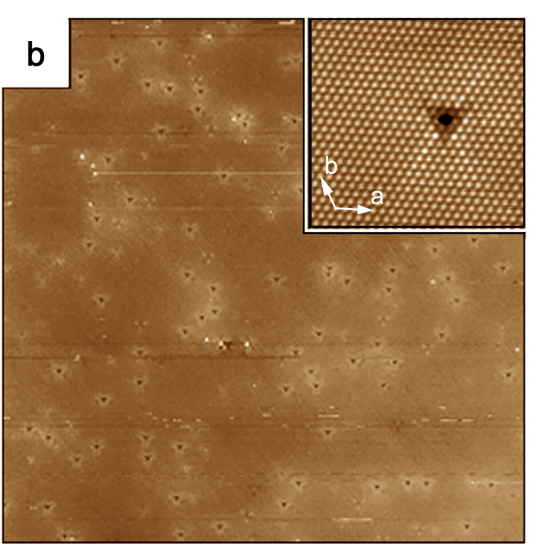

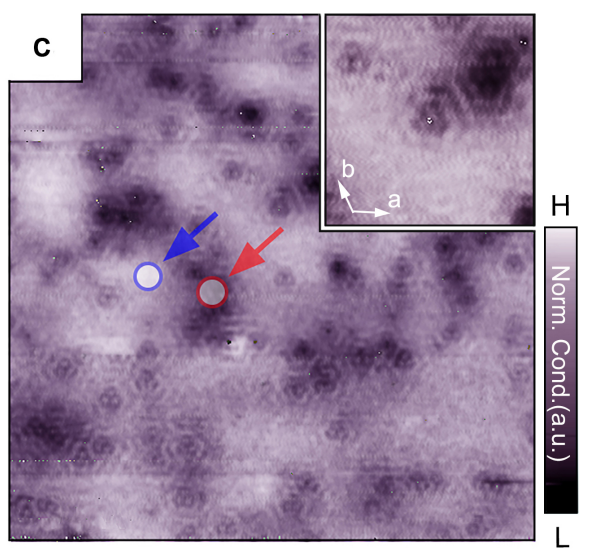

e

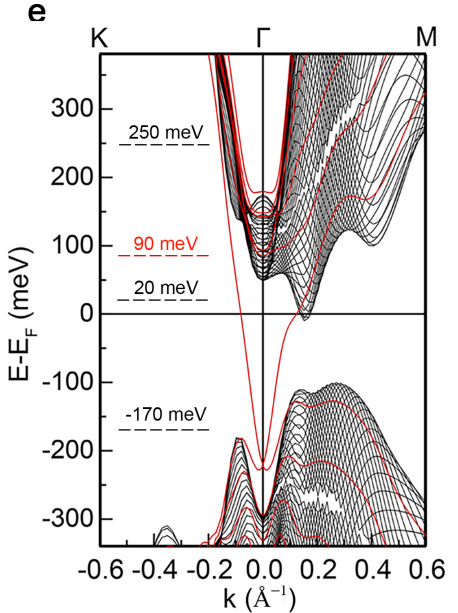

f
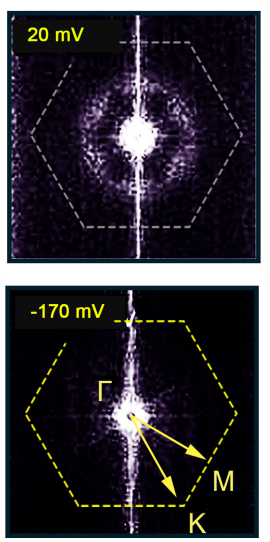
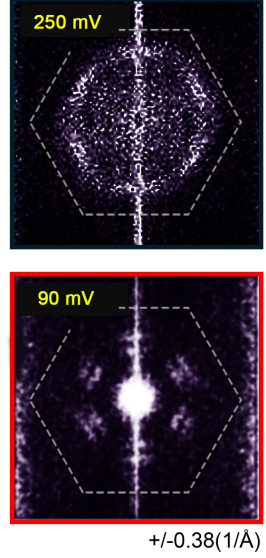

Figure 1: Electronic structure of $\mathrm{Bi}_{2} \mathrm{Te}_{3}$ and disorder. (a) Schematic top and side views on the structure of $\mathrm{Bi}_{2} \mathrm{Te}_{3}$. (b) $200 \times 200 \mathrm{~nm}^{2} \mathrm{STM}$ image of in situ cleaved $\mathrm{Bi}_{2} \mathrm{Te}_{3}$ crystal surface $\left(V_{\text {bias }}=200 \mathrm{mV}, I_{T}=100 \mathrm{pA}\right)$. Inset: $8 \times 8 \mathrm{~nm}^{2}$ zoom on a single defect. (c) Tunneling conductance $d I / d V$ map of the region in (b) acquired at the sample bias $V_{\text {bias }}=90 \mathrm{mV}$. Inset: $49 \times 49 \mathrm{~nm}^{2}$ zoom shows complex Friedel oscillation patterns due to scattering from several defects. (d) Local $d I / d V(V)$ spectra taken on a defect (red) and away from defects (blue) in the locations marked by arrows in (c). The vertical scale is adjusted to fit with the energy scale in (d). Dashed line: a linear extrapolation of Dirac branch to the Dirac point (DP) (see in the text). (e) Calculated (DFT) electronic structure of $\mathrm{Bi}_{2} \mathrm{Te}_{3}$. The Dirac cone is shown in red.(f) QPI patterns at four selected biases, corresponding to dashed lines in (d,e). $\Gamma-\mathrm{K}$ and $\Gamma-\mathrm{M}$ directions are marked with arrows. Dashed hexagonal contour of a fixed size accentuates the energy evolution of the scattering vector lengths. At $90 \mathrm{mV}$ the scattering vectors form six doublets aligned along $\Gamma-\mathrm{M}$ directions.

contours experience a perfect nesting, leading to a strong scattering at the nesting vectors $q_{n e s t}$. The QPI reflexes of this scattering channel are split; the splitting increases with reducing the quasiparticle lifetime. Further inclusion into the model of the realistic triangular shape of the scattering potential enhances the splitting and gives rise to the suppression of 
the scattering along $\Gamma-\mathrm{K}$ direction, in agreement with experimental observations.

The crystal structure of $\mathrm{Bi}_{2} \mathrm{Te}_{3}$ consists of Te-Bi-Te-Bi-Te quintuple atomic layers in $\vec{c}-$ crystallographic direction, Fig. 1a. The quintuple layers are linked to each other via van der Waals interaction, thus forming a lamellar easy-to-cleave material. Consequently, the outer $\left(\mathrm{Te}_{1}\right)$ and inner $\left(\mathrm{Te}_{2}\right)$ Te-atomic layers are not equivalent. Upon cleavage in UHV, macroscopically large atomically smooth $\mathrm{Te}_{1}$-terminated terraces are obtained and revealed in STM images, Fig. 1b. On top of atomic corrugation, triangle-shaped defects are observed, originating from atomic Te-vacancies (see inset in Fig. 1b). On the tunneling conductance map $d I / d V(x, y)$ of the same region acquired at the sample bias $V_{b i a s}=90 \mathrm{mV}$, the defects appear dark, reflecting a lower local electronic state density, Fig. 1c. Around defects, Friedel oscillations are observed; they overlap in regions of high defect density (inset in Fig. 1c).

Local $d I / d V(V)$ tunneling spectra taken at the position of a Te-vacancy (pointed by red arrow in Fig. 1c) look similar to those acquired away from impurities (blue arrow in Fig. 1c), as the respective curves in Fig. 1d demonstrate. Both present a low spectral weight in the window $-150 \mathrm{mV}$ to $0 \mathrm{mV}$, and a rapid raise at lower (higher) biases, consistent with previously reported results. ${ }^{39,40}$ This peculiar shape reflects the expected electronic structure of the material, Fig. 1e, where the bulk gap is crossed by topological surface bands (red curves) forming a Dirac cone. ${ }^{41}$ Since in the spectral window between $-100 \mathrm{mV}$ and $-50 \mathrm{mV}$ no influence of bulk states is expected, a linear extrapolation of $d I / d V(V)$ spectra to zero (dashed line in Fig. 1d) provides an estimate $-(210 \pm 30) \mathrm{meV}$ for the position of the Dirac point (DP) with respect to the Fermi level (zero-bias). ${ }^{21}$ The main difference between red and blue spectra in Fig. 1d is their slight relative distortion and a lateral shift at negative biases. The former reflects a slight redistribution of electronic states due to impurities; the latter points towards a slightly different energies of the DP near and far from the defects, of the order of $20 \mathrm{meV}$ (a more accurate estimation is difficult because of significantly different spectral weights of neighbouring bulk valence bands). Both cone distortion and variations of DP energy are even more evident in Figs. 2a-h where STS maps at various biases acquired in 

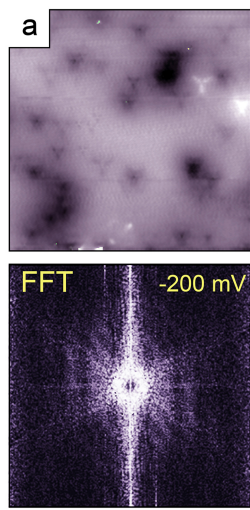

+/-0.38(1/Å)
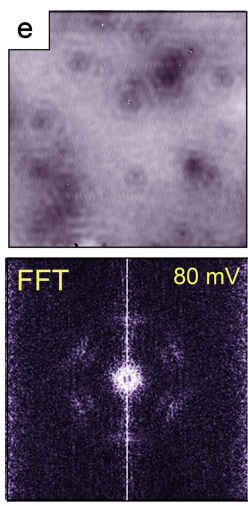

+/-0.38(1/Å)
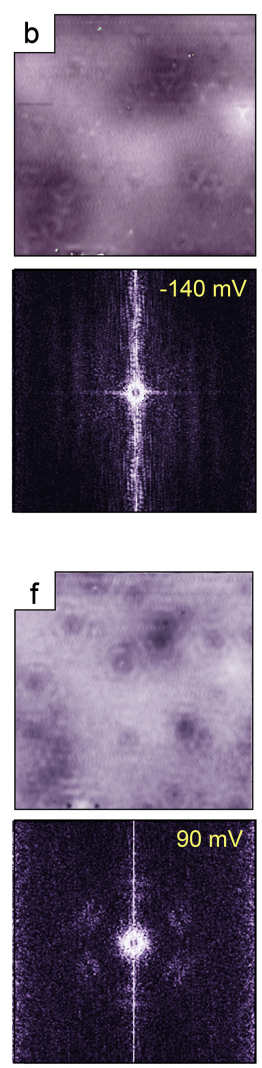
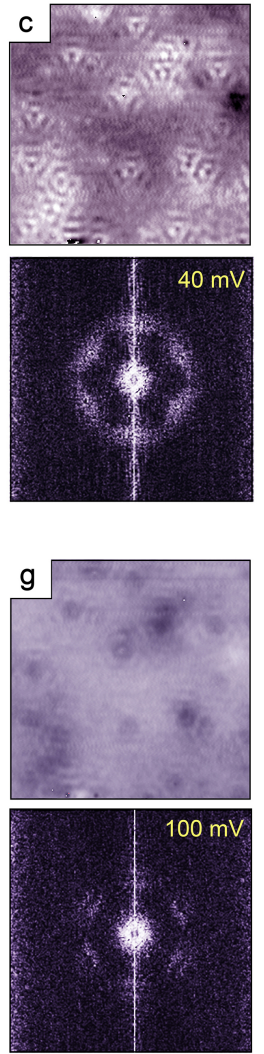
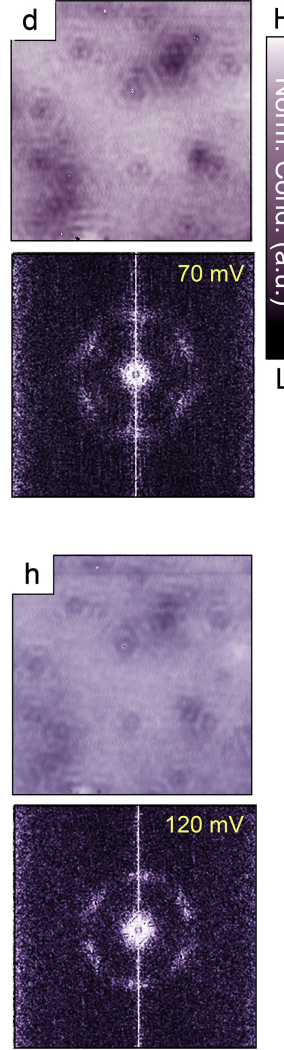
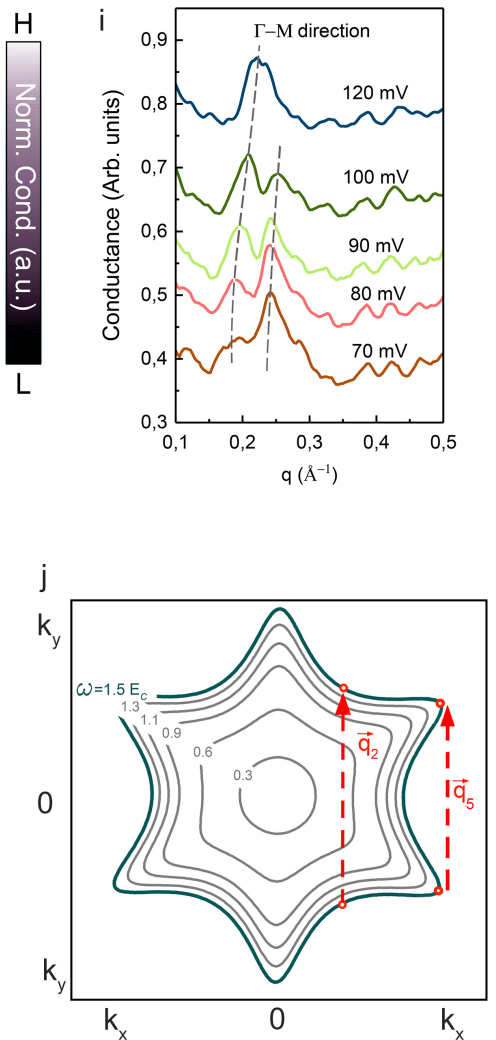

Figure 2: Quasiparticle scattering on atomic defects in $\mathrm{Bi}_{2} \mathrm{Te}_{3}$. (a)-(h) $75 \times 75 \mathrm{~nm}^{2} d I / d V$ tunneling conductance maps at different sample biases and their Fast Fourier Transformed patterns. (i) Evolution of the scattering peak amplitude in $\Gamma-\mathrm{M}$ direction with sample bias. (j) Constant energy contours in $k_{x}-k_{y}$ plane at different energies along with the spin texture of the corresponding electronic states. The scattering wave vector $q_{5}$ linking the nearest tips of the warped contour is presented by red arrow; a parallel vector $q_{2}^{28,29}$ - by a dashed red arrow.

the central part of the region Fig. 1b are presented. In all these maps, almost identical longrange variations of the contrast are observed, spatially correlated with the local concentration of defects.

The quasiparticle scattering is revealed in QPI patterns, Fig. 1f, which strongly depend on energy and nicely reflect the band structure of $\mathrm{Bi}_{2} \mathrm{Te}_{3}$, Fig. 1e. At $-170 \mathrm{mV}$, the scattering vectors are very short and form a spot around $\Gamma$ point, as expected for quasiaprticles close to $\mathrm{DP}$. At $20 \mathrm{mV}$, that is already $\simeq 230 \mathrm{meV}$ above the $\mathrm{DP}$, the vectors are much longer and form a distorted contour, due to hexagonal warping of the Dirac cone. At $90 \mathrm{mV}$, the 
hexagon disappears, and only six scattering spots along $\Gamma-\mathrm{M}$ directions remain, witnessing for the dominant role of warping in the scattering events. An intriguing feature here is a splitting of scattering spots in doublets, which we discuss below. At $250 \mathrm{mV}$ the scattering vectors form a halo, reflecting an additional contribution of the bulk conductance band in the scattering processes.

We now focus on Friedel oscillations observed around triangular defects. In Fig. 2 each STS map is presented along with its QPI pattern. At large negative biases, Figs. 2a,b, no Friedel oscillations are evident. In fact, at $V_{\text {bias }}=-(140-200) \mathrm{mV}$ the expected QPI scattering vectors are short, $q=0.01-0.03 \AA^{-1}$, and several effects wash the corresponding long-wave $(2 \pi / q=20-60 \mathrm{~nm})$ oscillations out: spatial variations of DP energy, short mean free path $l \sim 10 \mathrm{~nm}$ (we evaluate it later), escape of Dirac electrons to the bulk valence band, etc. At positive biases however, the oscillations are visible in the real space; the characteristic scattering vectors are revealed in the QPI patterns, Figs. 2c-h. The main scattering vectors are aligned in $\Gamma-\mathrm{M}$ directions, their length and amplitude evolve with the energy. The scattering vector splitting (SVS) is observed in the spectral window $60-120 \mathrm{mV}$; it is clearly visible in QPI patterns in Figs. 2e-g. Below and above this energy window, only six spots of scattering vectors are observed. The evolution of the SVS with energy is quite odd, Fig. 2 i. At $=70 \mathrm{mV}$, the QPI pattern is dominated by outer peaks; the amplitude of inner peaks is significantly lower. As the bias increases, the amplitude of outer peak lowers and that of the inner peak progressively increases. At $120 \mathrm{mV}$ the inner peak dominates, the outer one is barely visible. At yet higher bias, the outer peak vanishes completely, and only bright inner peaks are observed. The whole dynamics looks like a transfer of the scattering probability from one scattering channel to another. At first glance, it would be tentative to assign these channels to scattering vectors $q_{2}$ and $q_{5}$, Fig. $2 \mathrm{j}$, as previously suggested. ${ }^{28,29}$ The splitting of a QPI reflex was also observed at the $\mathrm{Au}(111)$ surface. $^{42}$ In the $\mathrm{Au}(111)$ surface one circle reflex comes from surface states and another circle comes from bulk states. At negative and low positive bias these reflexes coincide with each other, while at high bias bulk 
states reflex have smaller radii than reflex from surface states. This mechanism of reflex's doubling is inapplicable for our experiment since we observe splitting at biases placed in the bulk gap. Our calculations show that the physical origin of the SVS is essentially different. Due to warping, the constant energy contours get nested in a specific energy window. The highest scattering amplitude is accumulated precisely in the points matched by $q_{\text {nest }}$, see Fig. 3a. On the contrary, the scattering processes at vectors $q_{2}$ and $q_{5}$ are weak, because of the corresponding state densities are low. The key point is that the scattering reflex at $q_{\text {nest }}$ has a fine structure, with two close maxima, see top insert in Fig. 3a; the distance between the maxima is governed by the quasiparticle lifetime, as we show below. Thus, the SVS phenomenon is a new interference effect that occurs due to the superposition of two phenomena: partial nesting of the constant energy contours, and a relatively short quasiparticle lifetime, due to disorder. Specific triangular shape of the scattering potential of Te-vacancies only amplifies the SVS effect.

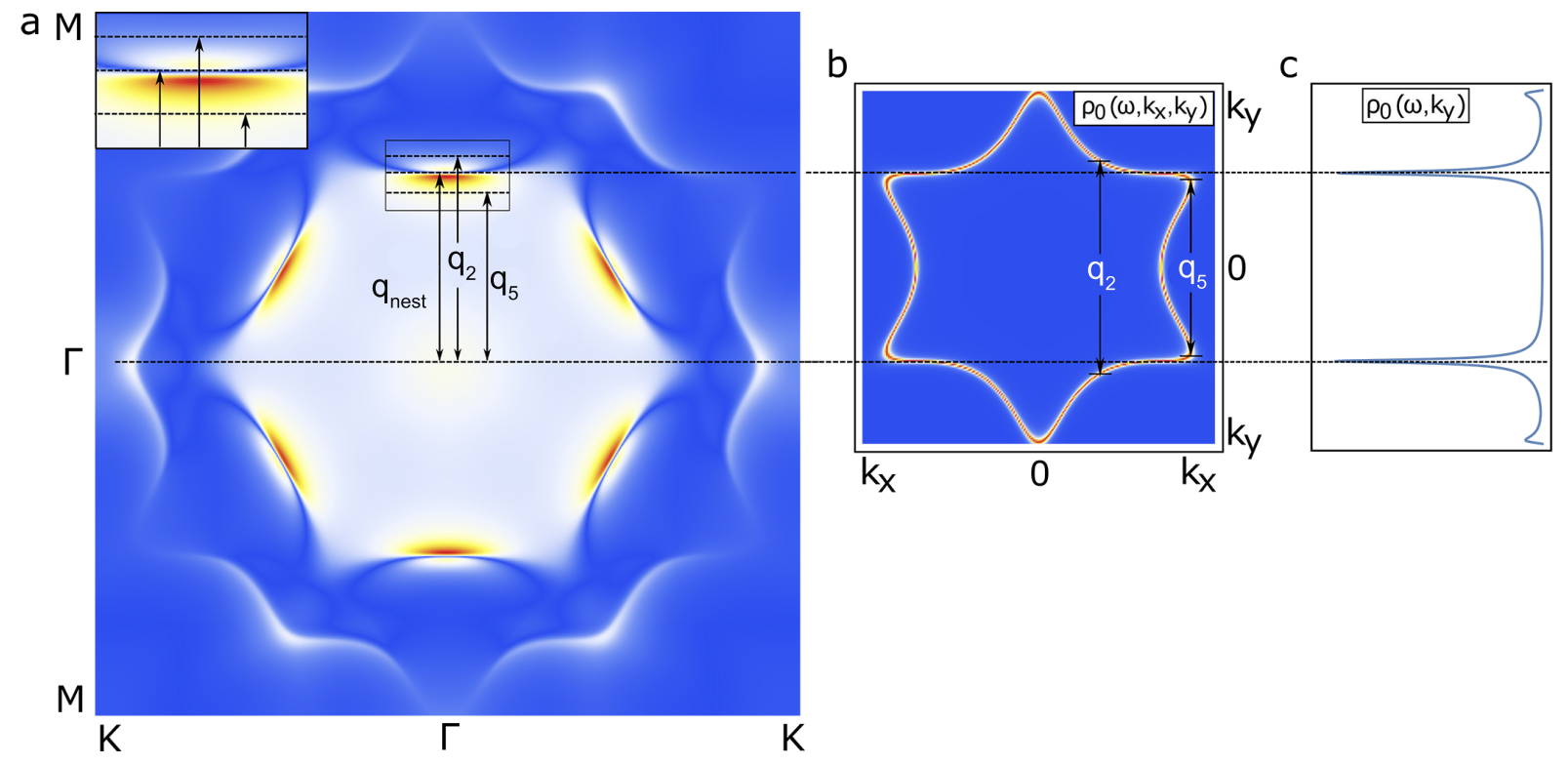

Figure 3: Scattering channels. (a) QPI picture for a single defect with scattering channels. Disorder broadening $\delta=0.03 E_{c}$ is relatively low and bias $\omega=1.25 E_{c}$. Vertical arrows show three possible scattering vectors $\mathbf{q}_{2}, \mathbf{q}_{5}$ and $\mathbf{q}_{\text {nest }}$. Black dashed line marks $q_{y}=0$ at the QPI. Other horizontal lines mark the height of scattering vectors. (b) DOS $\rho_{0}(\omega, \mathbf{k})=$ $\operatorname{Im}\left(\operatorname{Tr}\left[G_{0}(\omega, \mathbf{k})\right]\right)$. Vectors $\mathbf{q}_{2}$ and $\mathbf{q}_{5}$ are given. (c) Projection of the DOS on $k_{y}$ axis which is $\rho_{0}\left(\omega, k_{y}\right)=\int d k_{x} \rho_{0}(\omega, \mathbf{k})$. 
We start with presenting the results of calculations for a single point-like scatter potential $V_{\mathbf{k k}^{\prime}}=V_{0}$. In Fig. 4a we present QPI patterns generated for various energies and disorder strengths for a fixed $V_{0}=150 \mathrm{eV} \AA^{2}$. The energy range $1.1 E_{c}<\omega<1.6 E_{c}$ corresponds to the tunneling bias $65 \mathrm{mV}<V_{T}<185 \mathrm{mV}$ and thus overlaps with the bias window where SVS was experimentally observed. These calculations confirm that the main scattering occurs indeed along $\Gamma-\mathrm{M}$ directions, in agreement with the experimental data. Moreover, two interesting features are found. The first one is the fine structure of six scattering peaks. At low $\omega$, the inner part of the peak has a higher amplitude. As $\omega$ raises, the outer peak gets stronger, indeed consistent with the experimentally revealed dynamics. Note that the effect is robust with respect to disorder. The second feature is the dependence of the calculated QPI amplitude on disorder: at higher $\delta$ the scattering peaks have stronger amplitude and larger angular extensions.

To understand the physics behind the two features, let us remind that in the considered energy window the warping is already strong and the constant energy contour has a hexagonal shape. Near the tips, where warping vanishes, $\lambda k_{x}\left(k_{x}^{2}-3 k_{y}^{2}\right)=0$, the energy contour is flat. Such flat contour is nested with another flat region of the energy contour. Consequently, the main scattering vectors are those linking the nested flat parts with vector $\mathbf{q}_{\text {nest }}$, and the STS probes the resulting interference between these scatterings. Importantly, these are symmetric parts of the contour; one part can be translated into the other by a combination of the rotational $C_{3}$ and time-reversal symmetries.

We illustrate the role of nesting in Fig. 3a-c on which the scattering vectors $q_{2}, q_{5}$ and $q_{\text {nest }}$ are put on the calculated QPI pattern. The integrated along $k_{y}$ DOS has two strong peaks originating from flat parts of the constant energy contour - the manifestation of the nesting effect. The scattering channel between these two areas is described by $q_{n e s t}$. Remarkably, we found that exactly at $q_{\text {nest }}$ the reflex intensity is suppressed (seen as a thin blue line in Fig. 3a), while just above and just below two bright maxima exist (red and white spots in Fig. 3a). Thus, the reflex at $q_{\text {nest }}$ has a two-bump fine structure. The Green's function of 
a system may be written as $G=\sum_{n} \frac{|n\rangle\langle n|}{\epsilon-\epsilon_{n}+i \delta}$, where $|n\rangle$ and $\epsilon_{n}$ are $n$-th eigenfunction and eigenvalue of the system. ${ }^{43}$ For qualitative illustration we consider single point-like scatter in the first Born approach. ${ }^{44}$ The quasiparticle scattering density can be expressed in a form

$$
\rho(\mathbf{q}) \propto \operatorname{Im} \int \frac{\left|\left\langle\psi_{\mathbf{k}} \mid \psi_{\mathbf{q}+\mathbf{k}}\right\rangle\right|^{2}}{\left(\epsilon_{\mathbf{k}}+i \delta\right)\left(\epsilon_{\mathbf{k}+\mathbf{q}}+i \delta\right)} d^{2} \mathbf{k}
$$

where Im denotes the imaginary part of the integral; the energy is counted from $\omega, \epsilon_{k}=$ $E_{k}-\omega$, for simplicity. Due to partial nesting, the major scattering occurs at $q_{n e s t}$, and the integral can be substituted by the sum of two terms. One term comes from the scattering process $\mathbf{k} \rightarrow \mathbf{k}+\mathbf{q}_{\text {nest }}$ and other term comes from scattering between Kramers partners of this states. Since this two terms are equal, we omit one of them

$$
\rho(q) \simeq \rho\left(q_{n e s t}\right) \propto\left|\frac{\delta\left(\epsilon_{k}+\epsilon_{k+q_{n e s t}}\right)}{\left(\epsilon_{k} \epsilon_{k+q_{n e s t}}-\delta^{2}\right)^{2}+\delta^{2}\left(\epsilon_{k}+\epsilon_{k+q_{n e s t}}\right)^{2}}\right|
$$

Since $\epsilon_{k}=\epsilon_{k+q_{n e s t}}=0$, the density vanishes, $\rho\left(q_{\text {nest }}\right)=0$. This is a general result for the long wave scattering between symmetric points. It does not mean however that individual impurities do not produce Friedel oscillations around, as they locally break both translational and rotational symmetries. It just tells us that, at low scattering rates, one should expect the amplitude of QPI peaks exactly at $q_{\text {nest }}$ to be weak. The situation changes if $\delta \neq 0$ mixes up different energies around $\omega$ ( see Supplementary eq. 3). The scattering vectors $q$ may now differ from $q_{n e s t}$ by a disorder-dependent amount $\Delta q$, that is $q=q_{n e s t}+2 \Delta q$. Then, the quasiparticle scattering density becomes non-zero:

$$
|\rho(q)| \propto\left|\frac{\delta(\Delta q)^{2} \epsilon^{\prime \prime}(k)}{\left(\epsilon^{\prime}(k)^{2}(\Delta q)^{2}+\delta^{2}\right)^{2}+\delta^{2}(\Delta q)^{4} \epsilon^{\prime \prime}(k)^{2}}\right|,
$$

where $\epsilon^{\prime}(k)$ and $\epsilon^{\prime \prime}(k)$ are the first and the second derivatives of $\epsilon(k)$, respectively. The density Eq. 1 vanishes at $\Delta q \rightarrow 0$, as expected; its maximum value is reached at $\Delta q_{s v s}$ such that $\Delta q_{s v s}^{2}=\delta^{2} / \sqrt{\epsilon^{14}+\delta^{2} \epsilon^{\prime \prime 2}}$, that corresponds to doublets in QPI appearing in $\Gamma-\mathrm{M}$ 


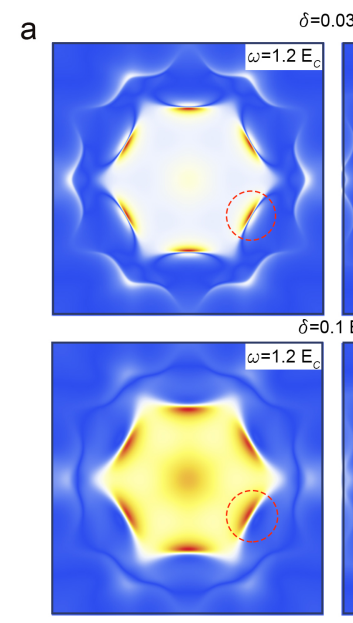

$\delta=0.03 \mathrm{E}_{c}$
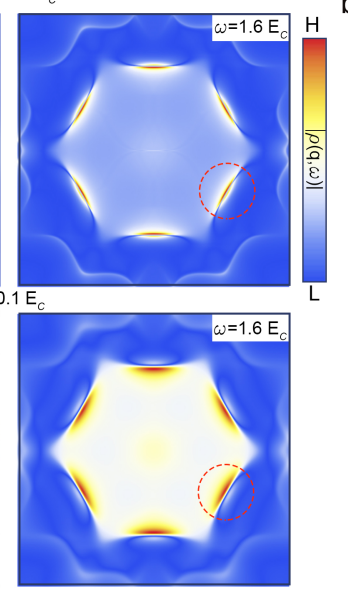
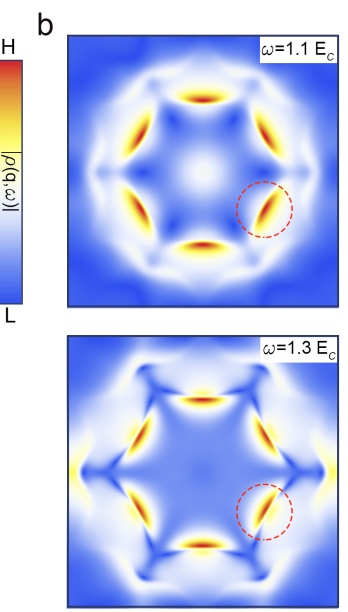
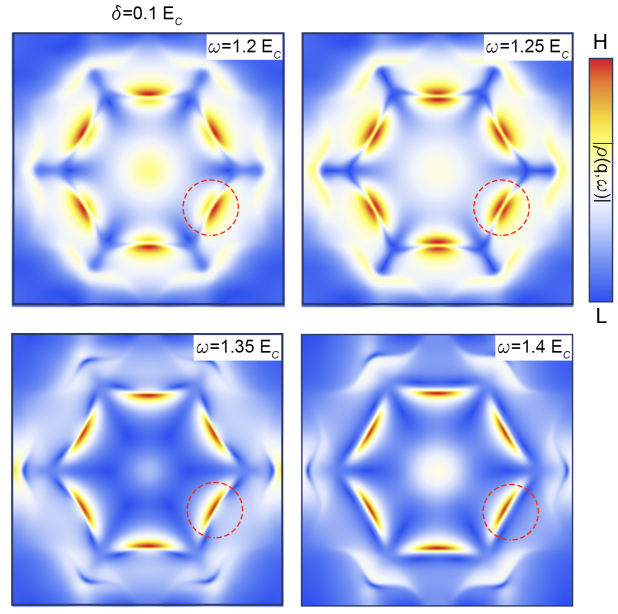

Figure 4: Calculated QPI patterns. Labels denote the considered scattering rates $\delta$ and energies $\omega$. (a) QPI due to the scattering on a single point defect. (b) Energy evolution of the QPI patterns due to the scattering on a triangular impurity. Red dashed circles focus on the region of interest.

directions at the wave vectors $q_{ \pm}=q_{\text {nest }} \pm \Delta q_{\text {svs }}$. Depending on relative weights of $\epsilon^{\prime}(k)$ and $\epsilon^{\prime \prime}(k)$, the distance between the maxima around $q_{\text {nest }}$ increases with increasing disorder as $\Delta q \propto \delta$ or $\Delta q \propto \sqrt{\delta}$. Thus, the disorder promotes the double-peak structure in QPI at the scattering vectors $q \simeq q_{\text {nest }}$ and makes the SVS experimentally observable at high enough $\delta$. Note however, that at too high $\delta$ Friedel oscillations are difficult to detect. The present case is an example of a general phenomenon: scattering between nested regions of the Fermi surface is split due to finite quasiparticle life-time arise from elastic scattering on impurities.

While our calculations indeed generate SVS, the absolute value of the obtained splitting $2 \Delta q_{\text {svs }}$ in Fig. 4a is tiny, significantly lower than the splitting observed in the experiment, Fig. 2. This pushed us to consider a more realistic shape of the scattering potential. The STM and density functional theory calculations ${ }^{35}$ demonstrate that in $\mathrm{Bi}_{2} \mathrm{Te}_{3}$ Te-vacancies form a triangular defect with the side about four lattice constants (see inset in Fig. 1b). It is then straightforward to model such a potential by a sum of the three scalar point potentials located at the vertices. ${ }^{32,35}$ The Fourier transform of this impurity potential is written as:

$$
V\left(k_{x}, k_{y}\right)=\frac{V_{0}}{3}\left(e^{-i k_{y} a \sqrt{3} / 3}+e^{i k_{x} a / 2+i k_{y} a \sqrt{3} / 6}+e^{-i k_{x} a / 2-i k_{y} a \sqrt{3} / 6}\right),
$$


where $a$ is the distance between point scatters in the triangle.

We found that using the three-vertex potential of the same total strength $V_{0}=150 \mathrm{eV} \AA^{2}$, as in the previous case of single point defect) results in stronger peaks in $\Gamma-\mathrm{M}$ directions and, remarkably, in a much stronger SVS. The finite size of the triangular defect $a$ suppresses scattering along $\Gamma-\mathrm{K}$ directions and make SVS phenomena more visible. The best match with the experimental data is obtained at $\delta=0.1 E_{c}$ and $a=18 \AA$. The latter is taken from, ${ }^{34}$ agrees with inset in Fig. 1b, and also matches, through the Bragg condition $q_{5} \sim \pi / a \simeq 0.2$ $\AA^{-1}$, the observed length of the scattering vectors in Fig. 2i. In Fig. $4 \mathrm{~b}$ the calculated QPI patterns reproduce all experimentally observed features. At low energies, six single peaks are visible. The peaks appear as stripes, similarly to the experimentally observed QPI pattern, Fig. 2d. At the energy $\omega>1.15 E_{c}$ the peaks form doublets, as they do in Fig. 2e-g. Such a double structure disappears at larger energies $\omega \sim 1.35 E_{c}$ and does not reappear at higher energies, as in Fig. 2h. The energy window where the splitting exists is therefore limited. Indeed, it requires, from one side, the warping to be strong enough, which is fulfilled at $\omega>E_{c}$. From the other side, the splitting vanishes at high energies when spectral non-linearities appear, that is when the kinetic energy due to the finite mass $\vartheta^{2} / r$ becomes comparable with $\omega$. Thus the splitting exists at $E_{c}<\omega<\vartheta^{2} / r$, which is a narrow window, since both warping and mass energies have close values $\sqrt{\vartheta^{3} / \lambda} \sim \vartheta^{2} / r$. It should be also mentioned that the previously predicted peaks along $\Gamma-\mathrm{K}$ directions ${ }^{28}$ do exist at larger momenta, Fig. 4; their amplitude is however low as compared to the peaks along $\Gamma-\mathrm{M}$ directions.

Finally, we estimate the scattering rate $\delta$ directly from experimental data and compare it with $\delta=0.1 E_{c}$ which was adjusted in Fig. 4b to fit with the experiment. Quasiparticle lifetime is mainly determined by three interactions: electron-electron, electron-phonon and electron-impurity. Due to the low temperature in our experiment, equal to $1.3 \mathrm{~K}$, scattering in the first and second channels is strongly suppressed. Thus we consider suggest that $\delta$ occurs due to averaging the Green's function over Gaussian randomly distributed point 
impurities, the scattering rate is: ${ }^{45}$

$$
\delta=n V_{0}^{2} \cdot \operatorname{Im} \int \frac{d^{2} \mathbf{k}}{(2 \pi)^{2}} G_{0}(\mathbf{k}, \omega),
$$

The defect density $n$ is estimated directly from the Fig. $1 \mathrm{~b}$ to $n \sim 10^{-2} k_{c}^{2}$. With the previously used $V_{0}=150 \mathrm{eV \AA} \AA^{2}$, we obtain $\delta \sim 20 \mathrm{meV}$, that is indeed not far from $0.1 E_{c}=$ $24 \mathrm{meV}$. As we can see, the scattering rate $\delta$ is quite large. Though, the corresponding mean free path $l=\vartheta \hbar / \delta \sim 10 \mathrm{~nm}$ looks reasonable. It is larger than $2 \pi / q_{5} \sim 3 \mathrm{~nm}$ and thus does not prevent Friedel oscillations from being experimentally observed.

\section{Methods}

The experiments were performed using Joule-Thompson low-temperature scanning tunnelling microscope Tyto ${ }^{T M}$ by SPECS $^{T M}$ GmbH operating in ultrahigh vacuum (base pressure less than $1 \times 10^{-10}$ Torr) at a temperature $1.3 \mathrm{~K}$. Single crystals of $\mathrm{Bi}_{2} \mathrm{Te}_{3}$ were cleaved in ultrahigh vacuum for in-situ STM/STS measurements. Topographic STM images were obtained in a constant-current mode. The STS was realized by acquiring local $I(V)(x, y)$ characteristics which were numerically differentiated to obtain the tunneling conductance $d I / d V(V)(x, y)$ maps.

\section{Acknowledgement}

VSS: I dedicate this work to my mother Stolyarova Larissa, who devoted her whole life to me. 23/06/1947-07/12/2020. We thank Wei-Cheng Lee for fruitful discussions. This work was supported by French-Russian (ANR - RSF) Research Grant "CrysTop" (RSF N20-42-09033). D.R. acknowledges COST Action CA16218 - Nanoscale Coherent Hybrid Devices for Superconducting Quantum Technologies. A.A.G. acknowledges support by the European Union H2020-WIDESPREAD-05-2017-Twinning project SPINTECH under Grant 
Agreement No. 810144. A.S.V. acknowledges support from the Academic Fund Program at the National Research University Higher School of Economics (HSE) in 2018-2019 (grant No. 18-01-0016) and the Russian Academic Excellence Project 5-100. D.A.K. and R.S.A. were supported by the Russian Scientific Foundation under Grant No 20-72-00030 and the Foundation for the Advancement of Theoretical Physics and Mathematics "BASIS". T.V.M. acknowledges support from Ministry of Education and Science of the Russian Federation (state task No. 0721-2020-0033).

\section{Supporting Information Available:}

Description of the material included.

\section{Author Contributions}

VSS and DR conceived and supervised the the project; VSS realized the STM/STS measurements with contributions from DR, SV and SP; TVM and EVC provided DFT calculations; DAK, RSA, DR, VS and VSS provided the explanation of the observed phenomena; DAK and RSA did numerical modelling with contributions from DR, VS and VSS; DR and VSS wrote the manuscript with the essential contributions from DAK and RSA along with the input from VS, HA, ASV, AAG and TC.

\section{References}

(1) Fu, L.; Kane, C. L.; Mele, E. J. Topological Insulators in Three Dimensions. Phys. Rev. Lett. 2007, 98, 106803.

(2) Hsieh, D.; Qian, D.; Wray, L.; Xia, Y.; Hor, Y.; Cava, R.; Hasan, M. Z. A topological Dirac insulator in a quantum spin Hall phase. Nature 2008, 452, 970-4.

(3) Zhang, H.; Liu, C.-X.; Qi, X.-L.; Dai, X.; Fang, Z.; Zhang, S.-C. Topological insulators 
in $\mathrm{Bi}_{2} \mathrm{Se}_{3}, \mathrm{Bi}_{2} \mathrm{Te}_{3}$ and $\mathrm{Sb}_{2} \mathrm{Te}_{3}$ with a single Dirac cone on the surface. Nature Physics 2009, 5, 438-442.

(4) Hasan, M. Z.; Kane, C. L. Colloquium: Topological insulators. Rev. Mod. Phys. 2010, 82, 3045-3067.

(5) Pi, S.-T.; Wang, H.; Kim, J.; Wu, R.; Wang, Y.; Lu, C.-K. New Class of 3D Topological Insulator in Double Perovskite. The Journal of Physical Chemistry Letters 2016, 8.

(6) Shun-Qing, S. Topological Insulators. Dirac Equation in Condensed Matters, 2nd ed.; 187; Springer Singapore, 2017; p 266.

(7) Tkachov, G. Topological Insulators: The Physics of Spin Helicity in Quantum Transport; Jenny Stanford Publishing, 2015; p 182.

(8) Chen, Y. L.; Analytis, J. G.; Chu, J.-H.; Liu, Z. K.; Mo, S.-K.; Qi, X. L.; Zhang, H. J.; Lu, D. H.; Dai, X.; Fang, Z.; Zhang, S. C.; Fisher, I. R.; Hussain, Z.; Shen, Z.-X. Experimental Realization of a Three-Dimensional Topological Insulator, Bi2Te3. Science 2009, 325, 178-181.

(9) Alpichshev, Z.; Analytis, J. G.; Chu, J.-H.; Fisher, I. R.; Chen, Y. L.; Shen, Z. X.; Fang, A.; Kapitulnik, A. STM Imaging of Electronic Waves on the Surface of $\mathrm{Bi}_{2} \mathrm{Te}_{3}$ : Topologically Protected Surface States and Hexagonal Warping Effects. Phys. Rev. Lett. 2010, $104,016401$.

(10) Henk, J.; Flieger, M.; Maznichenko, I. V.; Mertig, I.; Ernst, A.; Eremeev, S. V.; Chulkov, E. V. Topological Character and Magnetism of the Dirac State in Mn-Doped $\mathrm{Bi}_{2} \mathrm{Te}_{3}$. Phys. Rev. Lett. 2012, 109, 076801.

(11) Xia, Y.; Qian, D.; Hsieh, D.; Wray, L.; Pal, A.; Lin, H.-H.; Bansil, A.; Grauer, D.; Hor, Y.; Cava, R.; Hasan, M. Z. Observation of a large-gap topological-insulator class with a single Dirac cone on the surface. Nature Physics 2009, 5, 398-402. 
(12) Kuroda, K.; Arita, M.; Miyamoto, K.; Ye, M.; Jiang, J.; Kimura, A.; Krasovskii, E. E.; Chulkov, E. V.; Iwasawa, H.; Okuda, T.; Shimada, K.; Ueda, Y.; Namatame, H.; Taniguchi, M. Hexagonally Deformed Fermi Surface of the 3D Topological Insulator $\mathrm{Bi}_{2} \mathrm{Se}_{3}$. Phys. Rev. Lett. 2010, 105, 076802.

(13) Wang, Y. H.; Hsieh, D.; Pilon, D.; Fu, L.; Gardner, D. R.; Lee, Y. S.; Gedik, N. Observation of a Warped Helical Spin Texture in $\mathrm{Bi}_{2} \mathrm{Se}_{3}$ from Circular Dichroism AngleResolved Photoemission Spectroscopy. Phys. Rev. Lett. 2011, 107, 207602.

(14) Nomura, M.; Souma, S.; Takayama, A.; Sato, T.; Takahashi, T.; Eto, K.; Segawa, K.; Ando, Y. Relationship between Fermi surface warping and out-of-plane spin polarization in topological insulators: A view from spin- and angle-resolved photoemission. Phys. Rev. B 2014, 89, 045134.

(15) Eremeev, S. et al. Atom-specific spin mapping and buried topological states in a homologous series of topological insulators. Nature Communications 2012, 3, 635.

(16) Kou, L.; Ma, Y.; Sun, Z.; Heine, T.; Chen, C. Two Dimensional Topological Insulators: Progress and Prospects. The Journal of Physical Chemistry Letters 2017, 8.

(17) Freidel, J. Metallic alloys. Nuovo Cimento Suppl. 1958, 7, 287-311.

(18) Sprunger, P. T.; Petersen, L.; Plummer, E. W.; Lægsgaard, E.; Besenbacher, F. Giant Friedel Oscillations on the Beryllium(0001) Surface. Science 1997, 275, 1764-1767.

(19) Petersen, L.; Hofmann, P.; Plummer, E.; Besenbacher, F. Fourier Transform-STM: determining the surface Fermi contour. Journal of Electron Spectroscopy and Related Phenomena 2000, 109, 97 - 115.

(20) Zhang, T.; Cheng, P.; Chen, X.; Jia, J.-F.; Ma, X.; He, K.; Wang, L.; Zhang, H.; Dai, X.; Fang, Z.; Xie, X.; Xue, Q.-K. Experimental Demonstration of Topological 
Surface States Protected by Time-Reversal Symmetry. Phys. Rev. Lett. 2009, 103, 266803.

(21) Beidenkopf, H.; Roushan, P.; Seo, J.; Gorman, L.; Drozdov, I.; Hor, Y.; Cava, R.; Yazdani, A. Spatial Fluctuations of Helical Dirac Fermions on the Surface of Topological Insulators. Nature Physics 2011, 7.

(22) Sessi, P.; Otrokov, M. M.; Bathon, T.; Vergniory, M. G.; Tsirkin, S. S.; Kokh, K. A.; Tereshchenko, O. E.; Chulkov, E. V.; Bode, M. Visualizing spin-dependent bulk scattering and breakdown of the linear dispersion relation in $\mathrm{Bi}_{2} \mathrm{Te}_{3}$. Phys. Rev. B 2013, $88,161407$.

(23) Xu, Y.; Chiu, J.; Miao, L.; He, H.; Alpichshev, Z.; Kapitulnik, A.; Biswas, R.; Wray, L. Disorder enabled band structure engineering of a topological insulator surface. Nature Communications 2017, 8, 14081.

(24) Wang, J.; Li, W.; Cheng, P.; Song, C.; Zhang, T.; Deng, P.; Chen, X.; Ma, X.; He, K.; Jia, J.-F.; Xue, Q.-K.; Zhu, B.-F. Power-law decay of standing waves on the surface of topological insulators. Phys. Rev. B 2011, 84, 235447.

(25) Chen, M.; Jiang, Y.-P.; Peng, J.; Zhang, H.; Chang, C.-Z.; Feng, X.; Fu, Z.; Zheng, F.; Zhang, P.; Wang, L.; He, K.; Ma, X.-C.; Xue, Q.-K. Selective trapping of hexagonally warped topological surface states in a triangular quantum corral. Science Advances 2019,5 .

(26) Roushan, P.; Seo, J.; Parker, C.; Hor, Y.; Hsieh, D.; Qian, D.; Richardella, A.; Hasan, M. Z.; Cava, R.; Yazdani, A. Topological Surface States Protected From Backscattering by Chiral Spin Texture. Nature 2009, 460, 1106-9.

(27) Park, S. R.; Jung, W. S.; Kim, C.; Song, D. J.; Kim, C.; Kimura, S.; Lee, K. D.; Hur, N. Quasiparticle scattering and the protected nature of the topological states in a parent topological insulator $\mathrm{Bi}_{2} \mathrm{Se}_{3}$. Phys. Rev. B 2010, 81, 041405. 
(28) Zhou, X.; Fang, C.; Tsai, W.-F.; Hu, J. Theory of quasiparticle scattering in a twodimensional system of helical Dirac fermions: Surface band structure of a threedimensional topological insulator. Phys. Rev. B 2009, 80, 245317.

(29) Lee, W.-C.; Wu, C.; Arovas, D. P.; Zhang, S.-C. Quasiparticle interference on the surface of the topological insulator $\mathrm{Bi}_{2} \mathrm{Te}_{3}$. Phys. Rev. B 2009, 80, 245439.

(30) Liu, C.-X.; Qi, X.-L.; Zhang, H.; Dai, X.; Fang, Z.; Zhang, S.-C. Model Hamiltonian for topological insulators. Phys. Rev. B 2010, 82, 045122.

(31) Fu, L. Hexagonal Warping Effects in the Surface States of the Topological Insulator $\mathrm{Bi}_{2} \mathrm{Te}_{3}$. Phys. Rev. Lett. 2009, 103, 266801.

(32) Dai, J.; West, D.; Wang, X.; Wang, Y.; Kwok, D.; Cheong, S.-W.; Zhang, S. B.; Wu, W. Toward the Intrinsic Limit of the Topological Insulator $\mathrm{Bi}_{2} \mathrm{Se}_{3}$. Phys. Rev. Lett. 2016, $117,106401$.

(33) Unzueta, I.; Zabala, N.; Marín-Borrás, V.; Muñoz Sanjosé, V.; García, J. A.; Plazaola, F. Observation of a charge delocalization from Se vacancies in $\mathrm{Bi}_{2} \mathrm{Se}_{3}$ : A positron annihilation study of native defects. Phys. Rev. B 2016, 94, 014117.

(34) Kim, S.; Ye, M.; Kuroda, K.; Yamada, Y.; Krasovskii, E. E.; Chulkov, E. V.; Miyamoto, K.; Nakatake, M.; Okuda, T.; Ueda, Y.; Shimada, K.; Namatame, H.; Taniguchi, M.; Kimura, A. Surface Scattering via Bulk Continuum States in the 3D Topological Insulator $\mathrm{Bi}_{2} \mathrm{Se}_{3}$. Phys. Rev. Lett. 2011, 10\%, 056803.

(35) Netsou, A.-M.; Muzychenko, D. A.; Dausy, H.; Chen, T.; Song, F.; Schouteden, K.; Van Bael, M. J.; Van Haesendonck, C. Identifying Native Point Defects in the Topological Insulator $\mathrm{Bi}_{2} \mathrm{Te}_{3}$. ACS Nano 2020, 14, 13172-13179.

(36) Kohsaka, Y.; Machida, T.; Iwaya, K.; Kanou, M.; Hanaguri, T.; Sasagawa, T. Spin-orbit scattering visualized in quasiparticle interference. Phys. Rev. B 2017, 95, 115307. 
(37) Balatsky, A. V.; Vekhter, I.; Zhu, J.-X. Impurity-induced states in conventional and unconventional superconductors. Rev. Mod. Phys. 2006, 78, 373-433.

(38) Akbari, A.; Thalmeier, P. Full t-matrix approach to quasiparticle interference in noncentrosymmetric superconductors. The European Physical Journal B 2013, 86.

(39) Urazhdin, S.; Bilc, D.; Tessmer, S. H.; Mahanti, S. D.; Kyratsi, T.; Kanatzidis, M. G. Scanning tunneling microscopy of defect states in the semiconductor $\mathrm{Bi}_{2} \mathrm{Se}_{3}$. Phys. Rev. $B$ 2002, 66, 161306.

(40) Urazhdin, S.; Bilc, D.; Mahanti, S. D.; Tessmer, S. H.; Kyratsi, T.; Kanatzidis, M. G. Surface effects in layered semiconductors $\mathrm{Bi}_{2} \mathrm{Se}_{3}$ and $\mathrm{Bi}_{2} \mathrm{Te}_{3}$. Phys. Rev. B 2004, 69, 085313.

(41) Crowley, J.; Tahir-Kheli, J.; A., I. Accurate Ab Initio Quantum Mechanics Simulations of $\mathrm{Bi}_{2} \mathrm{Se}_{3}$ and $\mathrm{Bi}_{2} \mathrm{Te}_{3}$ Topological Insulator Surfaces. Journal of Physical Chemistry Letters 2015, 2015.

(42) Schouteden, K.; Lievens, P.; Van Haesendonck, C. Fourier-transform scanning tunneling microscopy investigation of the energy versus wave vector dispersion of electrons at the Au(111) surface. Phys. Rev. B 2009, 79, 195409.

(43) Kirkwood, J. In Mathematical Physics with Partial Differential Equations (Second Edition), second edition ed.; Kirkwood, J., Ed.; Academic Press, 2018; pp 153-185.

(44) Dutt, A.; Golubov, A. A.; Dolgov, O. V.; Efremov, D. V. Quasiparticle interference in multiband superconductors with strong coupling. Phys. Rev. B 2017, 96, 054513.

(45) D. Mahan, G. Many-Particle Physics. Physics of Solids and Liquids., 3rd ed.; Springer US, 2000; p 785 . 